Check for updates

Cite this: J. Mater. Chem. C, 2018, 6, 7012

Received 26th February 2018 Accepted 16th April 2018

DOI: $10.1039 / \mathrm{c} 8 \mathrm{tc} 00971 \mathrm{f}$

rsc.li/materials-c

\title{
Enhancing the sensitization efficiency of erbium doped organic complexes by heavy halogen substitution $\dagger$
}

\author{
J. X. Hu, (D) ${ }^{a}$ S. Karamshuk, ${ }^{a b}$ J. Gorbaciova, ${ }^{a} H$. Q. Ye, (D) ${ }^{a} H . L u, D^{c}$ Y. P. Zhang, ${ }^{a}$ \\ Y. X. Zheng, (D) ${ }^{d}$ X. Liang, ${ }^{d}$ M. Motevalli, ${ }^{b}$ I. Abrahams, (D) ${ }^{b}$ I. Hernández, (D) e \\ P. B. Wyatt (D) $*^{b}$ and W. P. Gillin (D) *af
}

\begin{abstract}
Lanthanide complexes with organic ligands have been of interest due to the possibility of sensitising lanthanide ions through the antenna effect. We have recently shown that the zinc(I) salt of 2-(3,4,5,6-tetrafluoro-2hydroxyphenyl)-4,5,6,7-tetrafluorobenzothiazole, $\mathrm{Zn}(\mathrm{FBTZ})_{2}$, can provide very high levels of sensitisation to organic erbium complexes whilst maintaining very high quantum yields for the erbium near-infra-red luminescence. We now report the spectroscopic properties of the zinc(II) salts of 2-(3,4,5,6-tetrafluoro-2hydroxyphenyl)-6-chloro-4,5,7-trifluorobenzothiazole and 2-(3,4,5,6-tetrafluoro-2-hydroxyphenyl)-6-bromo4,5,7-trifluorobenzothiazole. The substitution of a single chlorine atom into the chromophore is sufficient to double the sensitisation efficiency compared to the fully fluorinated chromophore. This result implies a $50 \%$ reduction in the pump intensity needed to achieve population inversion in the erbium ions.
\end{abstract}

\section{Introduction}

Near-infrared luminescent materials based on trivalent lanthanides such as $\mathrm{Nd}$, Er, and $\mathrm{Yb}$ have gained great attention in recent years due to their broad applications in laser and telecommunications systems, sensors and organic light-emitting diodes (OLEDs). ${ }^{1-3}$ Lanthanide ions display unique properties including narrow emission spectral width, and long luminescence lifetime. Erbium-doped materials emitting light at the wavelength of $1.5 \mu \mathrm{m}$ within the C-band of the optical telecommunication window, have been intensely investigated. ${ }^{4-6}$ However, like other

\footnotetext{
${ }^{a}$ Materials Research Institute and School of Physics and Astronomy, Queen Mary University of London, Mile End Road, London E1 4NS, UK. E-mail:w.gillin@qmul.ac.uk

${ }^{b}$ Materials Research Institute and School of Biological and Chemical Sciences, Queen Mary University of London, Mile End Road, London E1 4NS, UK. E-mail:p.b.wyatt@qmul.ac.uk

${ }^{c}$ State Key Laboratory of ASIC and System, SIST, Fudan University, Shanghai 200433, China

${ }^{d}$ State Key Laboratory of Coordination Chemistry, Nanjing National Laboratory of Microstructures, School of Chemistry and Chemical Engineering, Nanjing University, Nanjing 210093, China

${ }^{e}$ Dpto. CITIMAC, Facultad de Ciencias, Universidad de Cantabria, Avda. Los Castros, s/n 39005 Santander, Spain

${ }^{f}$ College of Physical Science and Technology, Sichuan University, Chengdu 610064, China

$\dagger$ Electronic supplementary information (ESI) available: Spectroscopic details. CCDC 1824389. For ESI and crystallographic data in CIF or other electronic format see DOI: 10.1039/c8tc00971f
}

lanthanides, erbium ions have very small absorption cross sections due to the partial forbiddance of $\mathrm{f}-\mathrm{f}$ electronic transitions. As a consequence, high power excitation is required to achieve signal amplification. The erbium emission can be enhanced by organic ligands through sensitization often referred to as the antenna effect. The most commonly accepted sensitization mechanism is that the absorbed light promotes molecules to the first excited singlet state of the ligand, followed by the population of the triplet state through intersystem crossing (ISC), and the energy is transferred from the triplet state to the erbium ion, which finally emits from the ${ }^{4} \mathrm{I}_{13 / 2}$ state. ${ }^{7,8}$ However, efficient vibrational quenching of erbium emission imposed by $\mathrm{OH}$ and $\mathrm{CH}$ bonds in the organic environment results in a major challenge for organic ligand design. ${ }^{9,10}$ Fluorination of the organic ligands has proven to be a successful approach and can improve the quantum efficiency of the erbium ions. Despite the relative success achieved by the fluorination of erbium-doped organic complexes, one of the problems with producing perfluorinated ligands and chromophores is the strongly electron-withdrawing effect of the fluorine atoms. ${ }^{11,12}$ Consequently, many of the organic complexes are not chemically or thermally stable. Previously, we proposed an idea to separate the chromophore function from that of producing a highly efficient organic environment for the erbium ion while creating an effective energy transfer between the chromophore and the erbium in a composite material. ${ }^{13}$ Erbium(III) tetrakis(pentafluorophenyl)imidodiphosphinate, ${ }^{11} \mathrm{Er}$ (F-TPIP) ${ }_{3}$ provides a long luminescence lifetime at $1.5 \mu \mathrm{m}$ for the encapsulated erbium ions, co-doped with a perfluorinated zinc-based organic chromophore, the zinc(II) 


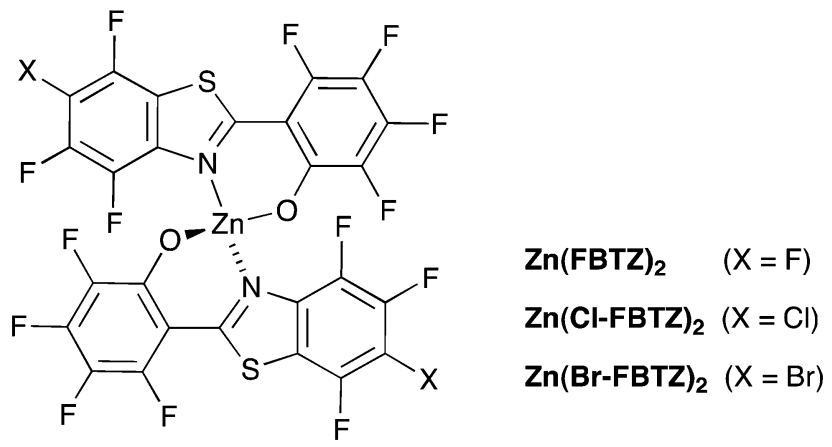

Fig. 1 Molecular structures of $\mathrm{Zn}(\mathrm{FBTZ})_{2}, \mathrm{Zn}(\mathrm{Cl}-\mathrm{FBTZ})_{2}$ and $\mathrm{Zn}(\mathrm{Br}-\mathrm{FBTZ})_{2}$ chromophores.

salt of 2-(3,4,5,6-tetrafluoro-2-hydroxyphenyl)-4,5,6,7-tetrafluorobenzothiazole, $\left(\mathrm{Zn}(\mathrm{FBTZ})_{2}\right)$ (Fig. 1). Efficient sensitization from $\mathrm{Zn}(\mathrm{FBTZ})_{2}$ to $\operatorname{Er}(\mathrm{F}-\mathrm{TPIP})_{3}$ has been observed, and a simple planar waveguide showing $3.3 \mathrm{~dB} \mathrm{~cm}^{-1}$ gain was fabricated based on the hybrid materials. ${ }^{13}$ This approach gives the potential to control the individual functions of the two molecules through design at a molecular scale. For example increasing the bulk of the erbium complex can fine tune the inter-ion distances in order to control self quenching whilst the incorporation of heavy atoms into the chromophore systems can enhance the intersystem crossing (ISC) to convert the singlet to triplet states. ${ }^{14-16}$ It is suggested that energy transfer from the triplet states to erbium ions is regarded as the most common pathway, however, it is unclear how the favoured triplet states affect the sensitisation effect. We have used chromophore molecules based on the original $\mathrm{Zn}(\mathrm{FBTZ})_{2}$ where the fluorine atom at the 6-position on each FBTZ ligand has been replaced by $\mathrm{Cl}$ or $\mathrm{Br}$. In this approach, the heavy-atom effect where halogen atoms are substituted into organic chromophores is utilized to improve the efficiency of spin-orbit coupling. Fluorescence quenching and a decrease of the phosphorescence lifetime are investigated to determine the enhanced ISC by introducing heavier halogen atoms and energy transfer. ${ }^{16,17}$ We then observe $\sim 50 \%$ improvements in the sensitisation efficiency with chlorine substitution compared to the fully fluorinated chromophore.

\section{Experiments}

The zinc phenoxide complexes $\mathrm{Zn}(\mathrm{Cl}-\mathrm{FBTZ})_{2}$ and $\mathrm{Zn}(\mathrm{Br}-\mathrm{FBTZ})_{2}$ (Fig. 1) were prepared by analogy with the synthesis of $\mathrm{Zn}(\mathrm{FBTZ})_{2} \cdot{ }^{18}$ Both complexes were characterised by elemental microanalysis, IR, NMR and high resolution mass spectrometry (see ESI $\dagger$ ); the structure of $\mathrm{Zn}(\mathrm{Br}-\mathrm{FBTZ})_{2}$ was confirmed by single crystal X-ray diffraction.

Co-doped films of Zn(FBTZ $)_{2}$ and $\operatorname{Er}(\text { F-TPIP })_{3}, \mathrm{Zn}(\text { Cl-FBTZ })_{2}$ and $\operatorname{Er}(\mathrm{F}-\mathrm{TPIP})_{3}, \mathrm{Zn}(\mathrm{Br}-\mathrm{FBTZ})_{2}$ and $\operatorname{Er}(\mathrm{F}-\mathrm{TPIP})_{3}$ were deposited onto glass substrates by chemical vapor deposition at a pressure of $\sim 10^{-7}$ mbar. An aluminum film with $100 \mathrm{~nm}$ thickness was deposited on top of the co-doped organic films to protect the material from degradation and to reflect the pump lasers back through the samples to increase absorption. Samples were fabricated with $\operatorname{Er}(\mathrm{F}-\mathrm{TPIP})_{3}$ concentrations of 9, 23, 43, 64, and
$80 \mathrm{~mol} \%$ and thicknesses of $66 \mathrm{~nm}, 100 \mathrm{~nm}, 180 \mathrm{~nm}, 350 \mathrm{~nm}$ and $750 \mathrm{~nm}$, respectively. An identical amount of the organic chromophores (50 nm thickness) was used in each film to ensure that the absorbance of chromophores was constant. Y(F-TPIP) 3 doped films (Y replacing the Er) with the same structure were also prepared for low-temperature photoluminescence measurements.

Absorption spectra were recorded with a Hitachi U-3000 spectrometer from organic chromophores dissolved in chloroform. The measurable range is from $190 \mathrm{~nm}$ to $900 \mathrm{~nm}$ and the wavelength measurement accuracy is $0.1 \mathrm{~nm}$. The emission spectra at room temperature were measured using a Jobin Yvon Horiba Triax 550 spectrometer connected with a Hamamatsu R5509-72 photomultiplier and extracted using a single-phase lock-in amplifier. The excitation spectra were obtained using a xenon arc lamp as light source coupled to a Jobin-Yvon Triax 180 spectrometer, while an Oxford Instrument Cryostat was used to perform low-temperature measurement on the samples. For the low temperature measurements the sample temperature was set at $80 \mathrm{~K}$ and the phosphorescence lifetime was obtained from the $407 \mathrm{~nm}$ laser excitation with a pulse frequency of $0.27 \mathrm{~Hz}$ and a pulse width of $50 \mathrm{~ms}$ to ensure complete decay of the triplet emission under low temperature.

\section{Results and discussion}

In Fig. 2(a) we show the absorption spectra of the organic chromophores measured in chloroform. Two broad absorption peaks centred at $\sim 295 \mathrm{~nm}$ and $\sim 407 \mathrm{~nm}$ can be observed. The intense absorption at $407 \mathrm{~nm}$ shows that these chromophores can be excited by commercially available diode lasers. The substituted chromophores exhibit slight red shifts in the absorption maxima relative to the fluorinated chromophore and the absorption maxima for the chlorinated and brominated chromophores are similar. To obtain the IR excitation spectra the emission intensity at $1.5 \mu \mathrm{m}$ was monitored whilst the excitation wavelength was scanned. The data were corrected for the changes in excitation intensity as a function of wavelength. An intense excitation band located at $\sim 410 \mathrm{~nm}$ for $\mathrm{Zn}(\mathrm{Cl}-\mathrm{FBTZ})_{2}$ and $\mathrm{Zn}(\mathrm{Br}-\mathrm{FBTZ})_{2}$, $\sim 420 \mathrm{~nm}$ for $\mathrm{Zn}(\mathrm{FBTZ})_{2}$ as can been observed from Fig. 2(b). Two very weak excitation peaks centred at $\sim 520 \mathrm{~nm}$ and $\sim 650 \mathrm{~nm}$ (see inset) also exist in the excitation spectra, which are due to the direct excitation of erbium ions through the ${ }^{4} \mathrm{I}_{15 / 2} \rightarrow{ }^{2} \mathrm{H}_{11 / 2}$ and ${ }^{4} \mathrm{I}_{15 / 2} \rightarrow{ }^{4} \mathrm{~F}_{9 / 2}$ transitions respectively. The dramatic difference of excitation intensity at $\sim 400 \mathrm{~nm}$ compared to the direct excitation into the ions indicates that sensitization through the chromophores is significantly more efficient than direct excitation of erbium ions.

The fluorescence spectra for the three chromophores at room temperature are displayed in Fig. 3. The chlorinated chromophore displays a small red shift relative to the fluorinated and brominated chromophores. Interestingly, the fluorescence bandwidth is slightly broadened by both chlorination and bromination. The broader fluorescence spectra and red shift may be beneficial to more efficient sensitization as the emission spectra of the chromophore have more spectral overlap with the absorption spectra of erbium ions. 

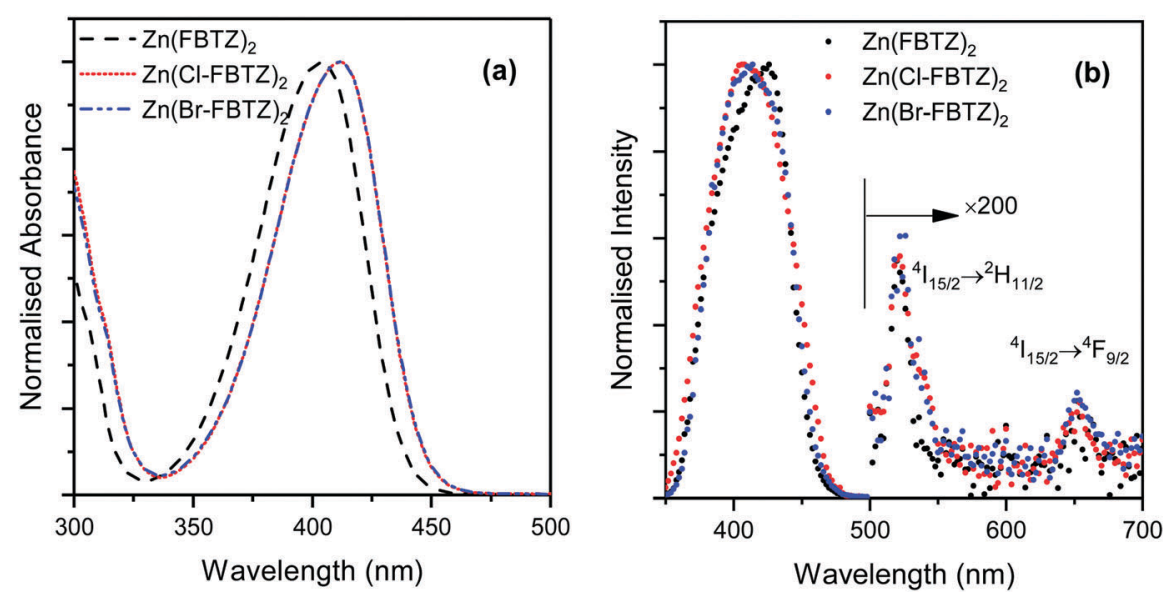

Fig. 2 (a) Absorption and (b) excitation spectra of $\mathrm{Zn}(\mathrm{FBTZ})_{2}, \mathrm{Zn}(\mathrm{Cl}-\mathrm{FBTZ})_{2}, \mathrm{Zn}(\mathrm{Br}-\mathrm{FBTZ})_{2}$ films co-doped with Er(F-TPIP) ${ }_{3}$ for the $1.532 \mu \mathrm{m}$ wavelength.

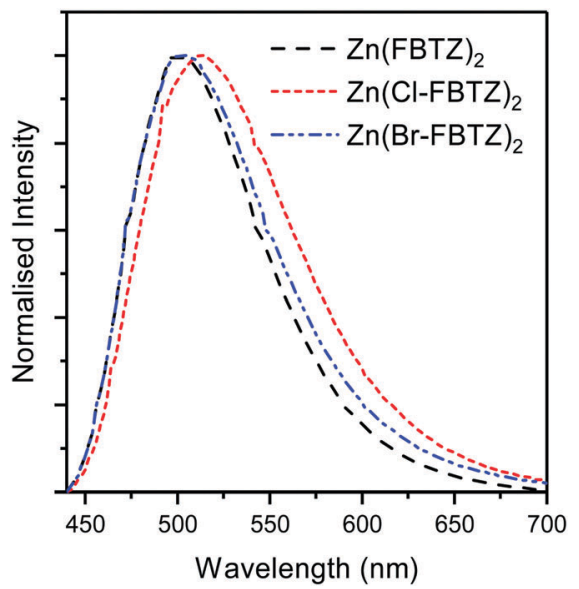

Fig. 3 Fluorescence spectra of $\mathrm{Zn}(\mathrm{FBTZ})_{2}, \mathrm{Zn}(\mathrm{Cl}-\mathrm{FBTZ})_{2}$, and $\mathrm{Zn}(\mathrm{Br}-$ $\mathrm{FBTZ})_{2}$.

The phosphorescence spectra and triplet lifetime of the chromophores were characterized at low temperature. A series of films containing a constant amount of ( $50 \mathrm{~nm}$ thick layer) chromophore with the same molar concentrations of Y(F-TPIP) ${ }_{3}$ as were used in the $\operatorname{Er}(\mathrm{F}-\mathrm{TPIP})_{3}$ doped films were also prepared. The Y(F-TPIP $)_{3}$ complex is transparent in the $350-1600 \mathrm{~nm}$ range and therefore, optically inert to the chromophores, which means any energy transfer process is excluded in the Y(F-TPIP $)_{3}$ codoped films.

Fig. 4 shows the emission spectra at $80 \mathrm{~K}$ for the three chromophores codoped into $80 \% \mathrm{Y}$ (F-TPIP $)_{3}$ normalised to the peak of the singlet emission. Two distinct emission peaks can be observed with peaks centred at $490 \mathrm{~nm}(2.53 \mathrm{eV})$ and $560 \mathrm{~nm}$ $(2.21 \mathrm{eV})$ which can be ascribed to the transition from the singlet and triplet states respectively. There are no obvious spectral shifts arising from the substitution of heavy halogen. The triplet emission intensity is greatly enhanced in samples doped with high Y(F-TPIP $)_{3}$ concentrations and the relative intensity ratio between the triplet and singlet increases from $\sim 0.5$ at $9 \% \mathrm{Y}(\mathrm{F}-\mathrm{TPIP})_{3}$ concentration to $\sim 1.6$ at $80 \% \mathrm{Y}(\mathrm{F}-\mathrm{TPIP})_{3}$. We ascribe this increase in the triplet emission to a reduction in

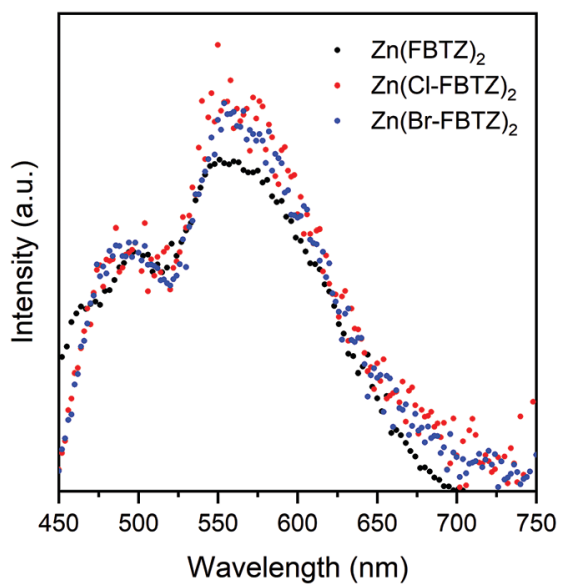

Fig. 4 Emission spectra for $80 \%$ Y (F-TPIP $)_{3}$ doped films measured at $80 \mathrm{~K}$ under the excitation of $407 \mathrm{~nm} \mathrm{CW}$ laser showing the triplet band peaking at $\sim 550 \mathrm{~nm}$.

triplet-triplet annihilation (TTA) as the chromophore concentration is reduced.

The reduction in TTA with increasing dilution is supported by transient PL measurements at $80 \mathrm{~K}$ and at an emission wavelength of $560 \mathrm{~nm}$ (the peak of the triplet emission) as a function of the chromophore concentration. Three distinct lifetimes can be measured. The shortest lifetime component $(\sim 10 \mathrm{~ns})$ is the singlet emission lifetime as the signals from the singlet and triplet emissions overlap. The two longer limetime components are of the order of $10 \mathrm{~ms}$ and $100 \mathrm{~ms}$ and are due to the triplets. The measured lifetime for each of these are shown in Fig. 5 as a function of Y(F-TPIP) $)_{3}$ concentration.

It can be seen that there is an increase in the triplet lifetime as the Y(F-TPIP $)_{3}$ concentration increases for each chromophore. This is to be expected if TTA is a significant source of triplet quenching in films with a high chromophore concentration. It can also be seen in Fig. 5 that the triplet lifetime decreases as the mass of the halogen in the 6 position increases. This can be ascribed to the fact that the substitution results in more efficient spin-orbit coupling due to the heavy 

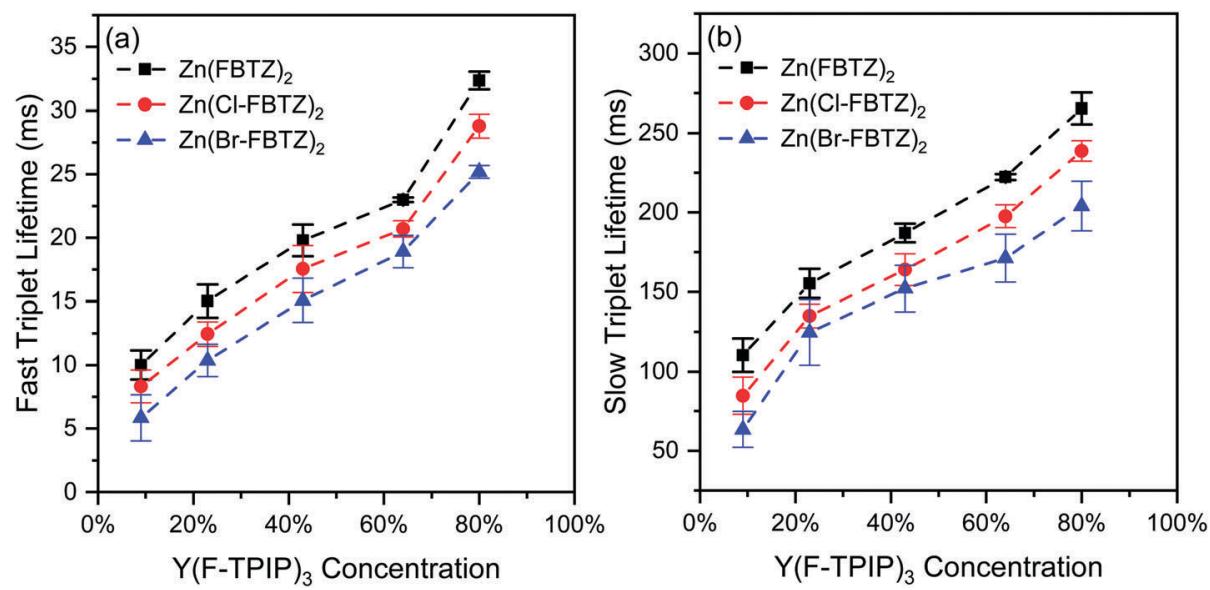

Fig. 5 The fast and slow triplet lifetime components for $\mathrm{Y}(\mathrm{F}-\mathrm{TPIP})_{3}$ codoped films measured at $80 \mathrm{~K}$ (excitation wavelength: $407 \mathrm{~nm}$, emission wavelength: $560 \mathrm{~nm}$ )

atom effect and more efficient ISC. Unfortunately, direct measurements of the ISC rate by measuring the rise time of the photoexcited triplet can not be performed in this system due to the strong spectral overlap between the singlet and triplet emissions.

For an equivalent set of films that were doped with $\operatorname{Er}(\text { F-TPIP })_{3}$ rather than $\mathrm{Y}(\mathrm{F}-\mathrm{TPIP})_{3}$ the triplet lifetimes are all significantly shorter which is indicative of energy transfer from the triplets to the $\mathrm{Er}^{3+}$ ions. In Fig. 6 we show the percentage reduction in the fast and slow triplets in the presence of $\operatorname{Er}(\mathrm{F}-\mathrm{TPIP})_{3}$ compared to $\mathrm{Y}(\mathrm{F}-\mathrm{TPIP})_{3}$. It can be seen that at low $\operatorname{Er}(\mathrm{F}-\mathrm{TPIP})_{3}$ concentrations the quenching of the triplets is very high (up to 90\%) and reduces as the $\operatorname{Er}$ (F-TPIP) ${ }_{3}$ concentration increases. As would be expected the slow (longer lifetime) triplets are more efficiently quenched by the $\mathrm{Er}^{3+}$ ions compared to the fast (shorter lifetime) triplets as there are fewer competing routes for decay. The energy transfer from $\mathrm{Zn}(\mathrm{FBTZ})_{2}$ and $\mathrm{Zn}(\mathrm{Br}-\mathrm{FBTZ})_{2}$ to the erbium is broadly similar although for the slow triplet the $\mathrm{Zn}(\mathrm{Br}-\mathrm{FBTZ})_{2}$ is slightly better. However, it can be seen that the triplet in $\mathrm{Zn}(\mathrm{Cl}-\mathrm{FBTZ})_{2}$ is significantly more efficient at coupling to $\operatorname{Er}(\mathrm{F}-\mathrm{TPIP})_{3}$ than for the other chromophores.
In addition to the improved energy transfer from the triplets into the erbium ions we also see significant reductions in the fluorescence intensity of the $\operatorname{Er}(\mathrm{F}-\mathrm{TPIP})_{3}$ doped films compared to those codoped with Y(F-TPIP $)_{3}$. For each chromophore the reduction in fluorescence intensity in the $80 \%$ co-doped films are $\sim 50 \%$ in the presence of erbium and there are no statistically significant differences between the chromophores.

In order to see how these observed effects translate into improved emission from the erbium ions we performed quantitative sensitisation measurements on all the samples. These were achieved by exciting a sample at two different wavelengths $407 \mathrm{~nm}$ and $655 \mathrm{~nm}$. The chromophores absorb light at $407 \mathrm{~nm}$ light efficiently, while the $655 \mathrm{~nm}$ light can only be absorbed by the erbium ions in the codoped films. The excitation light was directed vertically on the samples and the optical geometry was kept identical during the measurement. Each sample was excited as a function of pump power at each wavelength and the emission intensity of the erbium ions were subsequently modelled using a rate equation model. Full details of the approach have been reported previously. ${ }^{19}$
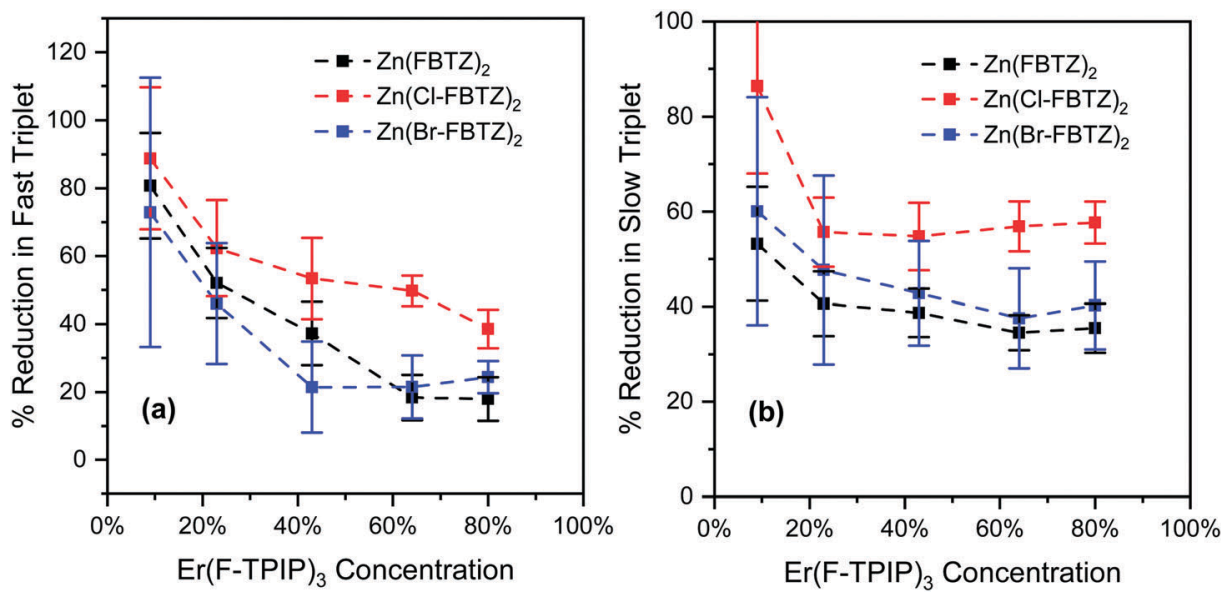

Fig. 6 The percentage reduction in the triplet lifetime components for $\operatorname{Er}(\mathrm{F}-\mathrm{TPIP})_{3}$ codoped films compared to Y(F-TPIP) 3 films, measured at $80 \mathrm{~K}$ under the excitation of $407 \mathrm{~nm}$ CW laser. 

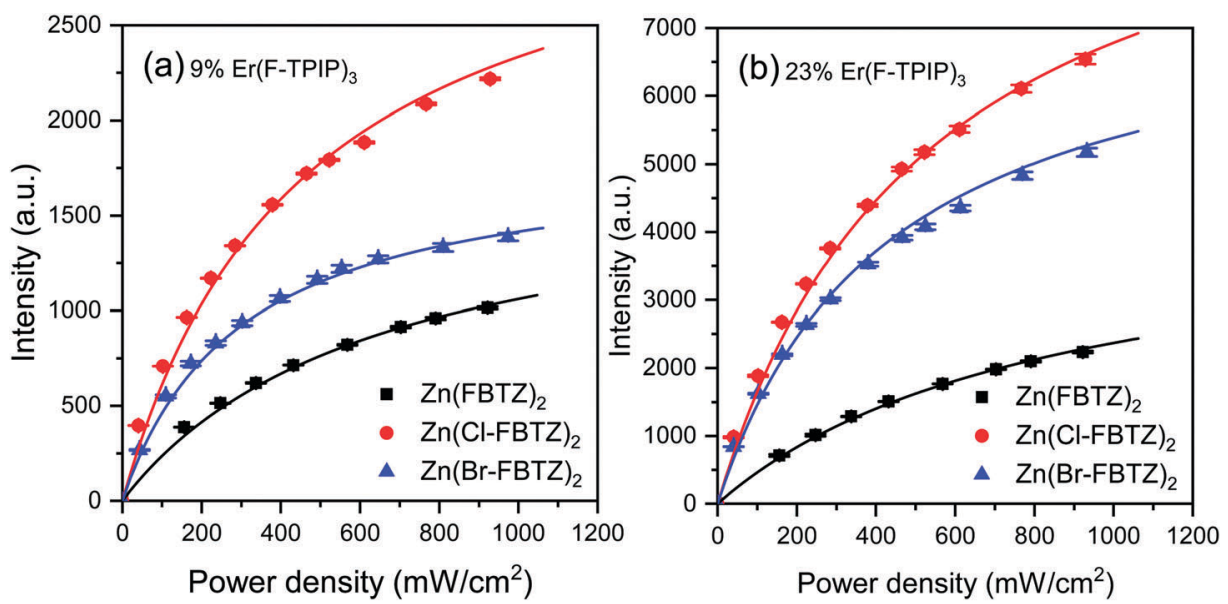

Fig. 7 Sensitisation data for the (a) $9 \%$ and (b) $23 \% \operatorname{Er}(\mathrm{F}-\mathrm{TPIP})_{3}$ doped films for each chromophore. The solid black line is the fit from the model.

In Fig. 7 we present the sensitisation data for the $9 \%$ and $23 \%$ doped films for each chromophore along with the best fit from the model. The full data set for each concentration can be found in the ESI. $\dagger$ At the lower erbium concentrations we find that the simple model provides an excellent fit to the data and allows us to determine a single sensitisation factor over all excitation power densities. At higher erbium concentrations the data starts to fall below the model predictions at high $407 \mathrm{~nm}$ pump intensities due to the contribution of Er-Er interactions as an additional quenching route. ${ }^{19}$

A summary of all the sensitisation data is presented in Fig. 8. It can be seen that for each chromophore the sensitisation is at a maximum for low erbium concentrations and reduces as the erbium concentration increases but tending to a single value at $\operatorname{Er}(\mathrm{F}-\mathrm{TPIP})_{3}$ concentrations greater than $\sim 40 \%$. It can clearly be seen that the $\mathrm{Zn}(\mathrm{Cl}-\mathrm{FBTZ})_{2}$ provides significantly enhanced $(\sim 40-60 \%)$ sensitisation compared to the $\mathrm{Zn}(\mathrm{FBTZ})_{2}$. The $\mathrm{Zn}(\mathrm{Br}-\mathrm{FBTZ})_{2}$ also shows enhanced sensitisation compared to $\mathrm{Zn}(\mathrm{FBTZ})_{2}(\sim 20-30 \%)$ although the sensitisation is less than for $\mathrm{Zn}(\mathrm{Cl}-\mathrm{FBTZ})_{2}$. This correlates with the reduction in triplet lifetimes in the presence of erbium shown in Fig. 6: for the slow triplets, i.e. those with fewer competing quenching routes,

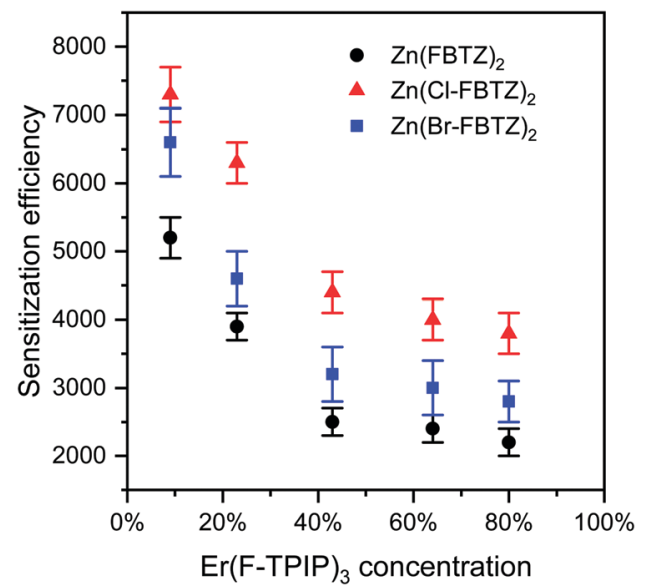

Fig. 8 Sensitisation efficiencies for the $\operatorname{Er}(\mathrm{F}-\mathrm{TPIP})_{3}$ doped samples.
$\mathrm{Zn}(\mathrm{Cl}-\mathrm{FBTZ})_{2}$ couple most efficiently followed by $\mathrm{Zn}(\mathrm{Br}-\mathrm{FBTZ})_{2}$ and finally $\mathrm{Zn}(\mathrm{FBTZ})_{2}$.

\section{Conclusions}

In conclusion, we have substituted fluorine atoms with chlorine and bromine atoms at position 6- on the perfluorinated chromophore $\mathrm{Zn}(\mathrm{FBTZ})_{2}$. Enhanced ISC after substitution is confirmed by triplet spectroscopy and lifetime measurements and the effect increases with the atomic number of the substituted atom. We observe that both singlets and triplet excitons couple efficiently with erbium and that for the triplet excitons the chlorinated chromophore has the most efficient coupling followed by the brominated and fluorinated. These results correlate with sensitisation measurements and show that it is possible to improve the energy transfer from a chromophore to erbium ions by modifying the excited state dynamics of the chromophore molecule.

\section{Author contributions}

J. X. H. prepared samples, collected and analysed the data. S. K., Y. X. Z., X. L., M. M., I. A. and J. G. contributed to the synthesis and characterization of the materials. H. Q. Y., H. L., J. G., Y. P. Z. and I. H. contributed to the data analysis and interpretation. P. B. W. directed the chemical synthesis programme. W. P. G. designed the study and wrote the paper. All authors discussed the results and commented on the manuscript.

\section{Conflicts of interest}

WPG, PBW and IH are all shareholders in Chromosol Ltd a spin-out company of QMUL working on the commercialisation of sensitised organic lanthanide complexes.

\section{Acknowledgements}

J. X. H., H. Q. Y., H. L. and Y. P. Z. were financially supported by the China Scholarship Council and Queen Mary University of 
London (QMUL). S. K. was supported by a Proof of Concept grant funded by QMUL and the EPSRC through the Silicon Photonics for Future Systems grant EP/L00044X. J. G. was financially supported by QMUL. I. H. acknowledges financial support from the Spanish Ministerio de Economia y Competitividad (Grant: MAT2016-80438-P). Y. Z. acknowledges financial support from the Major State Basic Research Development Program (2013CB922101) and NSFC (21371093). W. P. G. acknowledges financial support from EPSRC (EP/L020114/1 and EP/P007767/1) and from NSFC (61574095). We are grateful to the EPSRC UK National Mass Spectrometry Facility at Swansea University for spectra.

\section{Notes and references}

1 J. Kido and Y. Okamoto, Chem. Rev., 2002, 102, 2357-2368.

2 E. Desurvire, J. R. Simpson and P. C. Becker, Opt. Lett., 1987, 12, 888-890.

3 J. C. G. Bünzli, Acc. Chem. Res., 2006, 39, 53-61.

4 S. G. Roh, J. B. Oh, M. K. Nah, N. S. Baek, Y. I. Lee and H. K. Kim, Bull. Korean Chem. Soc., 2004, 25, 1503-1507.

5 A. Monguzzi, R. Tubino, F. Meinardi, A. O. Biroli, M. Pizzotti, F. Demartin, F. Quochi, F. Cordella and M. A. Loi, Chem. Mater., 2008, 21, 128-135.

6 R. J. Curry and W. P. Gillin, Appl. Phys. Lett., 1999, 75, 1380-1382.
7 J. C. G. Bünzli and S. V. Eliseeva, Lanthanide Luminescence, Springer, 2010, pp. 1-45.

8 Y. Hasegawa, Y. Wada and S. Yanagida, J. Photochem. Photobiol., C, 2004, 5, 183-202.

9 G. E. Buono Core, H. Li and B. Marciniak, Coord. Chem. Rev., 1990, 99, 55-87.

10 L. Winkless, R. H. C. Tan, Y. Zheng, M. Motevalli, P. B. Wyatt and W. P. Gillin, Appl. Phys. Lett., 2006, 89, 111115.

11 G. Mancino, A. J. Ferguson, A. Beeby, N. J. Long and T. S. Jones, J. Am. Chem. Soc., 2005, 127, 524-525.

12 L. Beverina, M. Crippa, M. Sassi, A. Monguzzi, F. Meinardi, R. Tubino and G. A. Pagani, Chem. Commun., 2009, 5103-5105.

13 H. Q. Ye, Z. Li, Y. Peng, C. C. Wang, T. Y. Li, Y. X. Zheng, A. Sapelkin, G. Adamopoulos, I. Hernández and P. B. Wyatt, Nat. Mater., 2014, 13, 382-386.

14 K. N. Solov'ev and E. A. Borisevich, Phys.-Usp., 2005, 48, 231-253.

15 M. R. Wright, R. P. Frosch and G. W. Robinson, J. Chem. Phys., 1960, 33, 934-935.

16 R. Bonnett, A. Harriman and A. N. Kozyrev, J. Chem. Soc., Faraday Trans., 1992, 88, 763-769.

17 H. Saigusa and T. Azumi, J. Chem. Phys., 1979, 71, 1408-1413. 18 Z. Li, A. Dellali, J. Malik, M. Motevalli, R. M. Nix, T. Olukoya, Y. Peng, H. Ye, W. P. Gillin and I. Hernández, Inorg. Chem., 2013, 52, 1379-1387.

19 J. X. Hu, S. Karamshuk, J. Gorbaciova, H. Q. Ye, H. Lu, Y. P. Zhang, Y. X. Zheng, X. Liang, I. Hernández, P. B. Wyatt and W. P. Gillin, Sci. Rep., 2018, 8, 3226-3234. 